\title{
Peripartum Cardiomyopathy (PPCM): dual case report and review of literatures
}

\begin{abstract}
Introduction: Peripartum cardiomyopathy (PPCM), unexplained heart failure is a rare complication of pregnancy that occurs at the end of pregnancy or early during the postpartum period. PPCM is a diagnosis of exclusion that is made after other possible etiologies have been eliminated. The presentation of PPCM is generally during the early postpartum period where the patient will typically present with heart failure symptoms; these symptoms are often mistaken for being part of the normal puerperal experience. Although there are many risk factors for the development of PPCM, the greatest risk factors include being of African descent, having multiple gestation pregnancies and advanced maternal age. The etiology of PPCM has never been completely understood. However, it has been postulated that it is related to persistent viral antigen exposure. The management guidelines of the patient with PPCM generally follow guidelines for the management of congestive heart failure resulting from other etiologies, with the exception that angiotensin-converting-enzyme inhibitors (ACE inhibitors) and angiotensin receptor blockers (ARBs), contraindicated during pregnancy, because of their teratogenic effects.
\end{abstract}

Case description: We present two cases of peripartum cardiomyopathy (PPCM) with the discussion of management and literature review.

Conclusion: Although the prognosis of PPCM is usually favorable, maternal mortality has been reported. Therefore, clinicians must remain vigilant to facilitate timely recognition of the peripartum complication. Serious nonfatal complications including cardiac dysrhythmias, progressive heart failure requiring heart transplantation, and thromboembolic events manifesting as cerebral vascular accidents and peripheral arterial embolism may occur. Early diagnosis and prompt treatment is associated with a decrease in morbidity and mortality associated with PPCM.

Keywords: arrhythmias, brain natriuretic peptide, cardiomyopathy, cardiovascular, dilated, echocardiogram, echocardiography, heart failure, left ventricular ejection fraction, LVEF, myocardial recovery, peripartum cardiomyopathy, PPCM, postpartum cardiomyopathy, PPCM, pregnancy complications, pregnancy, pregnancy-associated cardiomyopathy
Volume 4 Issue 2 - 2016

\author{
Shadi Rezai,' Richard Giovane BS, ${ }^{2}$ \\ Daniel Adams BS, ${ }^{2}$ Stephen LoBue BS, ${ }^{2}$ \\ Sri Gottimukkala,' Theodore Hale,' Ray \\ Mercado DO,' Cassandra E Henderson' \\ 'Department of Obstetrics and Gynecology, Lincoln Medical \\ and Mental Health Center, USA \\ ${ }^{2}$ George's University, USA
}

Correspondence: Cassandra E Henderson, Department of Obstetrics and Gynecology, Lincoln Medical and Mental Health Center, 234 East 149th Street, Bronx, NY, I045I, USA Email rezsha@sgu.edu

Received: January 12, 2016 | Published: February 05, 2016
Abbreviation: LV, left ventricular; LVEF, left ventricular ejection fraction; BNP, brain natriuretic peptide; $\mathrm{EF}$, ejection fraction; PND, paroxysmal nocturnal dyspnea; BiPAP, bilevel positive airway pressure; LTCS, low transverse cesarean section; ARBs, angiotensinreceptor blockers

\section{Introduction and background}

Peripartum cardiomyopathy (PPCM) is a form of dilated cardiomyopathy of unclear etiology, defined as heart failure secondary to left ventricular (LV) systolic dysfunction, ${ }^{1}$ which affects women without preexisting heart disease during the last month of pregnancy or during the first 5 months of postpartum. ${ }^{1-3}$ Patients with PPCM often show a severely reduced left ventricular ejection fraction (LVEF) at the time of diagnosis, but may recover a relevant proportion of their cardiac output. ${ }^{4}$ The incidence has been fully agreed upon but it estimated to be $0.025 \%$ to $0.03 \%$, i.e. $(1 / 4000)$ to $(1 / 3000)$ births respectively. ${ }^{5}$ Although PPCM shows a marked geographic and ethnic variation, it is most common in Africa and therefore among women of African descent. ${ }^{4-6}$ Most women present in the first month postpartum with typical heart failure symptoms such as dyspnea, lower extremity edema, and fatigue. ${ }^{3,4,7}$ PPCM can be particularly dangerous, as symptoms can be erroneously diagnosed as part of the normal puerperal process. ${ }^{8}$
The diagnosis of PPCM is based on the patient fulfilling 4 criteria described first by Pearson et al and Sliwa et al. ${ }^{6}$ Furthermore, the diagnosis can be aided by the finding of a significantly elevated serum Brain Natriuretic Peptide (BNP)., ${ }^{9} 10$ Although the role BNP plays in PPCM has not been described, it can be speculated that the low ejection fraction of the heart would further drive the production of BNP. $^{10}$

The etiology of PPCM is unclear; however there have been proposed mechanisms that speculated in the etiology of this disease. The first mechanism involves viral antigen exposure. ${ }^{1,2}$ Anti-inflammatory medications have been shown to decrease the disease process, thus favoring a viral etiology. Additionally, the presence of viral genome isolates has been found on myocardium tissue samples. Autoimmune process might play a role in the etiology of PPCM. Haghikia et al. ${ }^{11}$ demonstrated the possible role of auto antibodies against sarcomeric myosin and troponin I. ${ }^{11}$ They demonstrated a higher prevalence of PPCM in patients who had these two autoantibodies. ${ }^{11,12}$

Recent investigation suggests that elevated prolactin production may be responsible for the development of PPCM..$^{1,5,6}$ Prolactin is known to increase blood volume, decrease blood pressure and decrease angiotensin response. ${ }^{1}$ This would add further strain on the cardiovascular system and favor a state of increased blood volume. The use of bromocriptine in addition to standard PPCM has been shown to 
increase LVEF recovery as well as have better NYHA outcomes. ${ }^{5,6,13}$ Although an uncertain etiology, it has been agreed upon that PPCM is a result of either an excess of oxidative stress or increased expression of anti-angiogenic substances such as prolactin. ${ }^{14}$

Since PPCM can mimic symptoms of a normal puerperal process, attention should be placed on how to distinguish this pathology from a normal physiological process. Therefore, the aim of this report is to highlight possible pathology associated dyspnea in the postpartum period. $4,7,15,16$

\section{Presentation of the case I}

The Patient is a 38 year old African American P1102, with past medical history of hypertension, gestational diabetes and a former smoker of 4 cigarettes per day. Patient denied any cardiac disease in the childhood. Patient underwent emergency primary cesarean section at 36 weeks due to severe preeclampsia in an outside facility. Postpartum was complicated with preeclampsia and stroke (CVA) one week after delivery. The CVA episode presented with both a right sided residual upper extremity and lower extremity weakness and speech difficulty from cardiac thrombus source confirmed from an echocardiogram. An echocardiogram was done which showed a low ejection fraction (EF) and confirmed a LV thrombus, so she was placed on the following home medications: warfarin $7.5 \mathrm{mg}$, Lisinopril $2.5 \mathrm{mg}$ Daily, Coreg $3.125 \mathrm{mg}$ BID.

Relevant to the diagnosis of PPCM, was the patient's presentation to emergency department 6 weeks postpartum, complaining of acute Shortness of Breath (SOB) and chest pain. She stated that for the past 2 weeks, she had feelings of fatigue with decreased exercise tolerance (ET) to 1 block from 6 blocks with severe dyspnea. The patient sought medical treatment after developing an abrupt left sided chest pain, radiating to her back. The Patient also complained of lower extremity edema, and 2 days of orthopnea and Paroxysmal Nocturnal Dyspnea (PND). These symptoms were getting worse since delivery. Review of medications revealed that, the patient had prescription for Furosemide (Lasix) for her hypertension, however; she never took it.

A review of systems was negative for any cough, phlegm, fever, sick contacts or chest pain. The physical examination was remarkable only for bilateral basal crackles on the lung examination and pitting edema on the lower extremities. Table 1 shows selected labs and imaging for this patient.

Table I Selected Labs and Test Results for Patient I and 2

\begin{tabular}{|c|c|c|c|}
\hline $\begin{array}{l}\text { Labs/Test } \\
\text { performed }\end{array}$ & patient I results & Patient 2 results & Comments \\
\hline Troponin I & Troponin I Level: $0.058 \mathrm{ng} / \mathrm{mL}$ & $\begin{array}{l}\text { Troponin I level: } 0.18 \mathrm{ng} / \mathrm{mL}, 0.15 \mathrm{ng} / \mathrm{mL} \\
\text { (elevated but slowly trended down) }\end{array}$ & $\begin{array}{l}\text { Normal value for troponin I level is }<0.06 \mathrm{ng} / \\
\mathrm{mL}^{29}\end{array}$ \\
\hline Creatine Kinase (CK) & CK II 2 (U/L) & CK I 65 (U/L), CK & \\
\hline $\begin{array}{l}\text { Brain Natriuretic } \\
\text { Peptide (BNP) }\end{array}$ & BNP $1080 \mathrm{pg} / \mathrm{mL}$ & $\begin{array}{l}\text { BNP: } 479 \mathrm{pg} / \mathrm{mL} \text { (POD zero), } 385 / 404 \\
1419 \mathrm{pg} / \mathrm{mL} \text { (on POD I), } 552 \mathrm{pg} / \mathrm{mL} \text { (on } \\
\text { POD 2), I I } 90 \mathrm{pg} / \mathrm{mL} \text { (on POD 3), I I30 } \\
\text { pg/mL (on POD 4), 89I pg/mL (on POD } \\
\text { 5) }\end{array}$ & $\begin{array}{l}\text { Normal value for BNP is less than } 100 \mathrm{pg} / \mathrm{mL} \text {, } \\
\text { BNP levels of } 100-300 \mathrm{pg} / \mathrm{mL} \text { suggest heart } \\
\text { failure is present. BNP levels above } 300 \mathrm{pg} / \mathrm{mL} \\
\text { indicate mild heart failure. BNP levels above } \\
600 \mathrm{pg} / \mathrm{mL} \text { indicate moderate heart failure; BNP } \\
\text { levels above } 900 \mathrm{pg} / \mathrm{mL} \text { indicate severe heart } \\
\text { failure. }^{30}\end{array}$ \\
\hline
\end{tabular}

Electrocardiogram (EKG)

Echocardiogram enlargement No ischemic changes

On the day of admission:The $\mathrm{LV}$ is moderately dilated with global hypokinesis and severely depressed systolic functionsevere LV dysfunction. Fractional shortening (FS): 13 $\%$, Aortic root diameter: 2.7 $\mathrm{cm}$; EDV: $201 \mathrm{ml}$,Aortic root area: $5.9 \mathrm{~cm} 2$

ESV: 147 ml,ACS: $2.2 \mathrm{~cm}$; Ejection fraction (EF): $27 \%$, LA dimension: $4.2 \mathrm{~cm}$

Chest demonstrates the heart is enlarged in size. The mediastinal silhouette is unremarkable. There is no evidence of pleural effusion. There is no acute infiltrates (Figure I).
Sinus tachycardia with non specific $T$ wave abnormality

The left ventricle is enlarged with global hypokinesis and moderate to severely depressed systolic function. Elevated left ventricular (LV) filling pressures are present. Normal right ventricular size and systolic function. The left atrium is enlarged. Mild aortic insufficiency (AI) and tricuspid regurgitation (TR). Trace mitral regurgitation (MR). Left Ventricular Ejection fraction of $36 \%$.

Postpartum echocardiogram on II//2/15 showed Normal left ventricular ejection fraction of $63.8 \%$, with mild Tricuspid and mild pulmonic valvular regurgitations.

Disseminated multifocal patchy infiltrations in bilateral lung fields, with small bilateral pleural effusion (Figure 3).

Repeat Chest X-ray (after medical therapy) showed Resolution of pulmonary edema (Figure 4). 
Table Continued...

\begin{tabular}{|c|c|c|c|}
\hline $\begin{array}{l}\text { Labs/Test } \\
\text { performed }\end{array}$ & patient I results & Patient 2 results & Comments \\
\hline Chest CT Scan & $\begin{array}{l}\text { I. No CT evidence of acute } \\
\text { pulmonary embolus. } \\
\text { 2. Mild cardiomegaly and } \\
\text { interstitial edema without } \\
\text { evidence of pleural effusion. } \\
\text { 3. Subsegmental LEFT basilar } \\
\text { atelectasis } \\
\text { (Figure 2). }\end{array}$ & $\begin{array}{l}\text { No evidence of acute pulmonary } \\
\text { embolism identified. Small bilateral } \\
\text { pleural effusions are noted. Ground glass } \\
\text { opacities throughout the lungs. Interstitial } \\
\text { thickening/edema. History and findings } \\
\text { most consistent with pulmonary edema } \\
\text { (Figure 5). }\end{array}$ & \\
\hline Brain CT & Showed no acute change & Not Done & \\
\hline
\end{tabular}

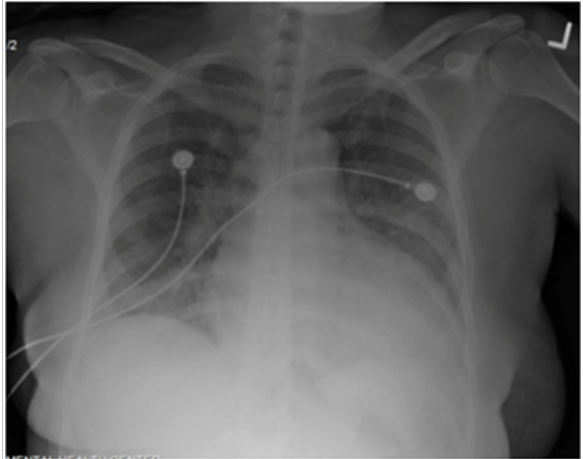

Figure I Chest $\mathrm{x}$-ray of Patient I: showing cardiomegaly with no pleural effusion.

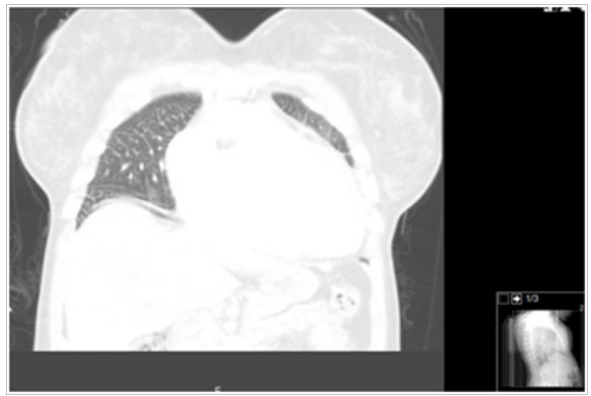

Figure 2 Chest CT-Scan of Patient I: showing cardiomegaly with no pleural effusion.

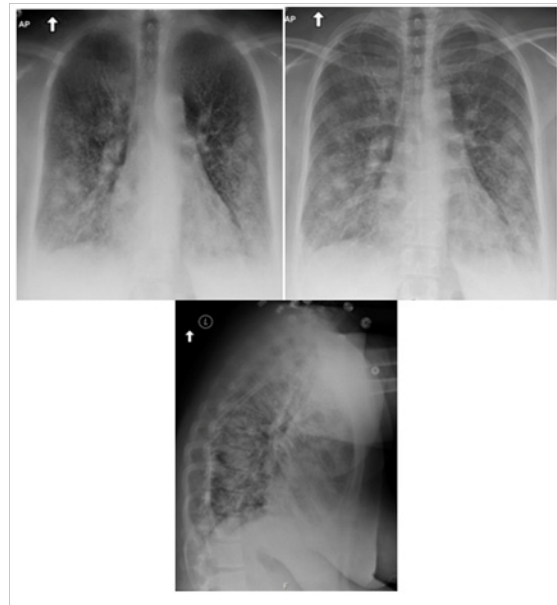

Figure 3 Patient 2 Chest $X$-ray: Disseminated multifocal patchy infiltrations in bilateral lung fields, with small bilateral pleural effusion.

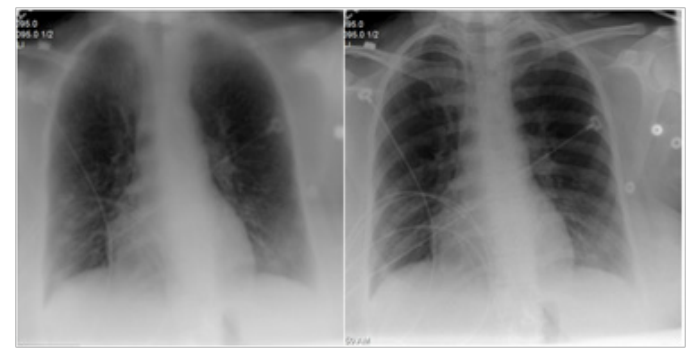

Figure 4 Patient 2 Chest X-ray (after medical therapy): Resolution of pulmonary edema: Previously seen bilateral interstitial infiltrates have resolved. No pleural effusion or pneumothorax. Heart is normal in size.

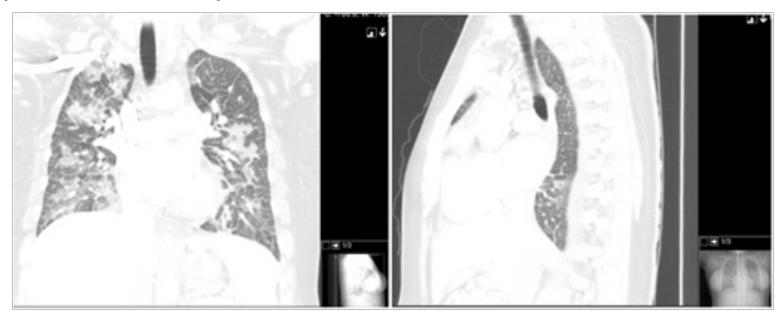

Figure 5 Patient 2 Chest CT Scan: No evidence of acute pulmonary embolism identified. Small bilateral pleural effusions are noted. Ground glass opacities throughout the lungs. Interstitial thickening/edema. History and findings most consistent with pulmonary edema.

Patient was admitted to MICU and was managed with Systolic heart failure protocol. patient received Lasix $40 \mathrm{mg}$, Oxygen, cardiac monitoring, Bilevel Positive Airway Pressure (BiPAP), due to respiratory distress as well as enoxaparin $40 \mathrm{mg}$ SQ and Lisinopril $2.5 \mathrm{mg}$ daily due to her hypertension and her history of CVA. The hospital course was uncomplicated, so she was discharged on Lasix $40 \mathrm{mg}$ PO, with follow up with her primary care provider and continuation of management.

\section{Presentation of the case 2}

The patient is a 21 years old Para 1001 with no past medical history who is a former smoker. She initially presented at 40 0/7 weeks gestation, consistent with a third trimester sonogram. The patient underwent a primary Low Transverse Cesarean Section (LTCS) for gestational hypertension (GHTN) with breech presentation with the development of preeclampsia without severe features. Postoperatively, patient completed a course of 24 hours magnesium sulfate for seizure prophylaxis due to preeclampsia. The patient subsequently developed a new onset of shortness of breath; diagnosed as pulmonary edema, on postoperative day four and was admitted to MICU for further medical management of preeclampsia induced postpartum cardiomyopathy and severe pulmonary edema. The patient denied ever had any 
heart problems, she was never was diagnosed with any heart valvar abnormality. A review of systems was negative for any cough, phlegm, fever, sick contacts or chest pain. Patient noted to have bilateral crackles on lung examination and bilateral lower extremities pitting edema. Table 1 shows selected labs and imaging for this patient.

While in the MICU, the patient received Labetalol $200 \mathrm{mg}$ IV drip, Digoxin IV, Nitrate drip and Lasix $40 \mathrm{mg}$ IVP. Lasix improved the patient's shortness of breath. She was discharged home on postoperative day 7 with Lasix $40 \mathrm{mg}$ PO daily, Coreg $6.25 \mathrm{q} 12$ hrs, Lisinopril $20 \mathrm{mg}$ daily, Digoxin $0.125 \mathrm{mg}$ daily and with closed follow up with cardiology and gynecology clinic.

Postpartum echocardiogram on 11/12/15 showed Normal left ventricular ejection fraction of $63.8 \%$, with mild Tricuspid and mild pulmonic valvular regurgitations.

\section{Discussion}

These 2 cased demonstrate some of the clinical presentation of PPCM including hypoxia, an elevated BNP level, cardiomegaly on chest radiographs and lower ejection fraction shown by echocardiography. To make a timely diagnosis it is important to be aware of the similarities and differences between PPCM and normal physiologic changes of pregnancy (Table 2). It is critical that PPCM be considered during the peripartum evaluation of a female., ${ }^{4,9}$

Although the etiology of PPCM is not fully understood, there have been proposed mechanisms of the disease which include viral etiology, autoimmune or increased prolactin. ${ }^{1,2,11}$ The cause of this disease may involve all of these etiologies however it has been agreed upon that PPCM is characterized by a state of increased oxidative stressors and increased expression of anti-angiogenic molecules.

There has been an association between maternal age, parity, hypertension and ethnicity with PPCM., ${ }^{7,15}$ Majority of patients with PPCM present at the age of 30 years and older ${ }^{7,15}$ therefore the second case described is one of interest as the patient is 21 years old and provides an example of PPCM occurring below the age of 30. The incidence of PPCM is most prominent in African American patients for reasons that are unclear. ${ }^{15}$ Patient's with PPCM also have an underlying diagnosis of hypertension which can make delay the diagnosis of PPCM and make management of the disease more difficult. ${ }^{7,14,15}$ Lastly, multifetal pregnancies have a strong association with the development of PPCM. ${ }^{10,15}$ Umazume et al. speculate that multifetal pregnancies increase gestational weight, which leads to volume expansion and increase the risk of cardiac overload and strain on the heart. ${ }^{10}$

Since there is an overlap of presenting features between PPCM and other hypertensive pathologies such as preeclampsia ${ }^{14,17,18}$ such as shortness of breath, fatigue and swelling ${ }^{8}$ it is important to be mindful of PPCM and how to diagnose it. Transthoracic echocardiography enables differentiation of heart failure with preserved ejection fraction, commonly observed in women with preeclampsia, from that with peripartum cardiomyopathy in which a reduced ejection fraction is more common. Table 2 demonstrates some of the findings when differentiating between PPCM versus some of the other differential diagnosis.

The diagnosis of PPCM must fulfill all four criteria following criteria. ${ }^{19}$

\section{All 4 of the following}

\section{Classic:}

a. Development of cardiac failure in the last month of pregnancy or within the first 5 months of postpartum

b. b. Absence of a determinable etiology for cardiac failure.

c. c. Absence of known heart disease prior to the last month of pregnancy.

\section{Additional:}

a. Left ventricular systolic dysfunction demonstrated by classic echocardiographic criteria such as depressed shortening fraction or ejection fraction [2]. Strict echocardiographic indication of left ventricular dysfunction:

b. Ejection fraction (EF) $<45 \%$ and/or

c. Fractional shortening $(\mathrm{FS})<30 \%$ (Fractional shortening $=$ (Enddiastolic dimension - End-systolic dimension) / End-diastolic dimension)

d. End-diastolic dimension (EDD) $>2.7 \mathrm{~cm} / \mathrm{m} 2$

e. The approach to providing patient care in PPCM has evolved in order to isolate clinical abnormalities about the patient. Certain tests are recommended which include the following:

\section{Diagnostic testing of PPCM: ${ }^{1,2}$}

A. Complete family history, to identify possible familial association

B. Serum tests

C. Complete blood cell count with differential

D. Creatinine and urea levels

E. Electrolyte levels, including magnesium and calcium

F. Levels of cardiac enzymes, including troponin

G. Level of B-type natriuretic peptide (BNP) and/or N-terminal pro-B-type natriuretic protein

H. Liver function tests (LFT's) and level of thyroid-stimulating hormone (TSH)

I. Chest radiograph

J. Electrocardiogram (EKG)

K. Transthoracic echocardiogram

L. Cardiac magnetic resonance imaging (MRI) and/or endomyocardial biopsy (when indicated). ${ }^{1}$ Magnetic resonance images can be used to measure global and segmental contraction and identify inflammatory processes. ${ }^{1}$

Table 2 Signs and symptoms in peripartum cardiomyopathy (PPCM) vs. normal pregnancy, PE and preeclampsia ${ }^{4,26,33}$

\begin{tabular}{lllll}
\hline & Pregnancy & Severe Preeclampsia & $\begin{array}{l}\text { Peripartum } \\
\text { Cardiomyopathy } \\
\text { (PPCM) }\end{array}$ & $\begin{array}{l}\text { Pulmonary Embolism Upper Respiratory } \\
\text { (PE) }\end{array}$ \\
\hline Mechanism & Physiologic & Diastolic Dysfunction & Systolic Dysfunction & Obstruction \\
Tachycardia & Tachycardia & $+/-$ & Tachycardia & Tachycardia \\
\hline
\end{tabular}


Table Continued...

\begin{tabular}{|c|c|c|c|c|c|}
\hline & Pregnancy & Severe Preeclampsia & $\begin{array}{l}\text { Peripartum } \\
\text { Cardiomyopathy } \\
\text { (PPCM) }\end{array}$ & $\begin{array}{l}\text { Pulmonary Embolism } \\
\text { (PE) }\end{array}$ & $\begin{array}{l}\text { Upper Respiratory } \\
\text { Infection (URI) }\end{array}$ \\
\hline S3 Heart Sound & Yes & Yes (as part of pregnancy) & S3 Heart Sound & S3 Heart Sound & NO \\
\hline Hypertension & NO & Yes & NO & $+1-$ & NO \\
\hline Fatigue & Fatigue & Fatigue & Fatigue & Fatigue & Fatigue \\
\hline Cough & NO & Usually not & Cough & $+/-$ & Cough \\
\hline Chest Pain & $+/-($ GERD $)$ & $+/-$ & Chest Pain & Chest Pain & $+/-$ \\
\hline Dyspnea & Dyspnea & Dyspnea & Dyspnea & Dyspnea & $+1-$ \\
\hline $\begin{array}{l}\text { Dyspnea on Exertion } \\
\text { (DOE) }\end{array}$ & $+1-$ & $+1-$ & DOE & DOE & $+1-$ \\
\hline Dyspnea (PND) / & Rare & NO & PND/Orthopnea & PND/Orthopnea & NO \\
\hline $\begin{array}{l}\text { Orthopnea } \\
\text { Rales }\end{array}$ & NO & $+1-$ & Rales & Rales & $+1-$ \\
\hline Hepatosplenomegaly & NO & NO & Hepatosplenomegaly & NO & NO \\
\hline Edema & Edema & Edema & Edema & Edema & NO \\
\hline Elevated Liver Enzymes & $+1-$ & Yes & NO & NO & NO \\
\hline Thrombocytopenia & $+1-$ & Yes & NO & NO & NO \\
\hline
\end{tabular}

PPCM has a multifactorial origin, therefore the clinical outcome varies based on time of diagnosis and how the patient is managed. ${ }^{1}$ In some cases, the patient improves dramatically with medical therapy however other in other instances, chronic heart failure from persistent ventricular dysfunction develops. The approach for treating PPCM mirrors that of treating congestive heart failure. This involves first restricting fluid and salt intake and then giving beta-blockers along with diuretics ${ }^{4-7}$ with the clinical goal to reduce the workload of the heart. The selection of diuretics is important as well. Angiotensin converting enzyme inhibitors (ACE-I) and angiotensin-receptor blockers (ARBs) are contraindicated in pregnancy and are therefore avoided. ${ }^{4,7}$ Spironolactone has been shown to be estrogenic in the rat model ${ }^{7,19}$ and as a precaution is not used during pregnancy. Metoprolol is the beta-blocker of choice due to its successful use during pregnancy but other beta-blockers such as atenolol are avoided due to known instances of fetal growth retardation. ${ }^{7,20}$ Lastly, hydralazine can be used during pregnancy to reduce after load and has been shown to have favorable outcomes. ${ }^{7,20}$ Diuretics must be used cautiously both during and after pregnancy as they can produce volume contraction which can lead to placental insufficiency. ${ }^{19,20}$

The use of bromocriptine in patients with PPCM has been a proposed treatment. ${ }^{20}$ Hilfiker-Kleiner et al. ${ }^{21}$ demonstrated a favorable outcome in patients given two doses of $2.5 \mathrm{mg}$ of bromocriptine daily for 6 weeks. It was found that these patients had a significant increase of LVEF by $31 \%$ as compared to the control group which was $9 \%$. The use of bromocriptine is limited to gestational use only as this medication suppresses lactation.

Patients with PPCM are at increased risk for thrombus formation. Therefore, general standard precautions and anticoagulation therapy is advised for patients that are high risk, such as those that are immobile or with severe LV dysfunction which favors thrombus formation. ${ }^{4,5}$ In addition, physical activity should be encouraged according to patients' tolerance of symptoms. ${ }^{22,23}$

In about $50 \%$ of patients, the ejection fraction normalizes after medical intervention. As a last resort, if medical treatments are not successful, heart transplantation is done. ${ }^{4}$ Progression of the PPCM requiring heart transplantation is described in $4 \%$ and death in $9 \%$ at a two years follow up. ${ }^{3}$ Other studies showed a much higher mortality rate such as $15 \%$ or $32 \%$ at 6 months. ${ }^{24}$ Survivors of PPCM have $30 \%$ risk of relapse ${ }^{4}$ and significant decrease of left ventricular function ${ }^{25}$ in subsequent pregnancies with reported $55 \%$ mortality and morbidity rates associated with recurrence in subsequent pregnancy. ${ }^{26}$ One explanation would be that patients who entered the subsequent pregnancy with abnormal systolic function, without making a complete recovery from the first PPCM in prior pregnancy. ${ }^{27}$

Since complete recovery from a relapse is very $\operatorname{rare}^{28,29}$ and due to increase chance of relapse in the next pregnancy, a second pregnancy is usually not recommended for patients with history of PPCM, which puts both mother and baby at great risk. ${ }^{30}$

\section{Conclusion}

PPCM is a rare disease, which can have devastating consequences if not diagnosed and treated early. The development of clinical guidelines and timely diagnosis has improved the lowered mortality rates associated with PPCM over the past 10 years due to advances in medical therapy for heart failure and use of implantable defibrillators. ${ }^{31}$ Management of PPCM includes the standard treatment of heart failure with reduced ejection fraction with special considerations for gravid as and nursing women. ${ }^{32}$

\section{Acknowledgments}

Special thanks to Ms. Judith Wilkinson, Medical Librarian, from Lincoln Medical and Mental Health Center Science Library for assistance in finding the reference articles.

\section{Conflicts of interest}

The authors declare there is no conflict of interests.

\section{Funding}

None.

\section{References}

1. Johnson-Coyle L, Jensen L, Sobey A. American Heart Association, Peripartum cardiomyopathy: review and practice guidelines. Am J Crit Care. 2012;21(2):89-98.

2. Groesdonk HV, Dinse-Lambracht A, Doblanzki W, et al. Unrecognized peripartum cardiomyopathy: Case series and comprehensive review of the literature. Applied Cardiopulmonary Pathophysiology. 2009;13(3):237-242. 
3. Cemin R, Janardhanan R, Daves M. Peripartum cardiomyopathy: an intriguing challenge. Case report with literature review. Curr Cardiol Rev Nov. 2009;5(4):268-272.

4. Wang M. Peripartum cardiomyopathy: case reports. Perm J. 2009; 13(4):42-45.

5. Capriola M. Peripartum cardiomyopathy: a review. Int $J$ Womens Health. 2012;5:1-8.

6. Meyer GP, Labidi S, Podewski E, et al. Bromocriptine treatment associated with recovery from peripartum cardiomyopathy in siblings: two case reports. J Med Case Rep. 2012;4:80.

7. Davis M, Duvernoy C. Peripartum cardiomyopathy: current knowledge and future directions. Womens Health (Lond Engl). 2015;11(4):565-573.

8. Patel H, Berg M, Barasa A, et al. Symptoms in women with Peripartum Cardiomyopathy: A mixed method study. Midwifery. 2016;32:14-20.

9. Kumari A, Singh S, Singh S, et al. Peripartum Cardiomyopathy: A Review of Three Case Reports. Journal ofSouth Asian Federation of Obstetrics and Gynecology. 2012;4(3):164-166.

10. Umazume T, Yamada T, Yamada S, et al. Peripartum cardiomyopathy in a woman with preeclampsia with twin pregnancy. BMJ Case Rep. $2014 ; 2014$

11. Haghikia A, Kaya Z, Schwab J, et al. Hilfiker-Kleiner D, Evidence of autoantibodies against cardiac troponin I and sarcomeric myosin in peripartum cardiomyopathy. Basic Res Cardiol. 2015;110(6):60.

12. Lockwood C. ACOG task force on hypertension in pregnancy-A step forward in management. Contemporary OB/GYN. 2013.

13. Dhesi S, Savu A, Ezekowitz J, et al. Diabetes during pregnancy and the risk of peripartum cardiomyopathy: A population-level analysis of 213,058 women. Canadian Journal of Cardiology. 2015;31(10):S292S293.

14. Hilfiker-Kleiner D, Haghikia A, Nonhoff J, et al. Peripartum cardiomyopathy: current management and future perspectives. Eur Heart J. 2015;36(18):1090-1097.

15. Elkayam U. Clinical characteristics of peripartum cardiomyopathy in the United States: diagnosis, prognosis, and management. J Am Coll Cardiol. 2011;58(7):659-670.

16. McNamara DM, Elkayam U, Alharethi R, et al. Clinical Outcomes for Peripartum Cardiomyopathy in North America: Results of the IPAC Study (Investigations of Pregnancy-Associated Cardiomyopathy). J Am Coll Cardiol. 2015;66(8):905-914.

17. Belen E, Tipi FF, Helvaci A, et al. Concurrent early-onset peripartum cardiomyopathy in a preeclampsia patient with acute pulmonary edema. Intern Med. 2015;54(8):925-927.
18. Ntusi NB, Badri M, Gumedze F, et al. Pregnancy-Associated Heart Failure: A Comparison of Clinical Presentation and Outcome between Hypertensive Heart Failure of Pregnancy and Idiopathic Peripartum Cardiomyopathy. PLoS One. 2015;10(8):e0133466.

19. Cardona-Guarache R, Kron J. Treatment of Peripartum Cardiomyopathy: A Call to Action. Can J Cardiol. 2015;32(12):1418-1420.

20. Desplantie O, Tremblay-Gravel M, Avram R, et al. The Medical Treatment of New-Onset Peripartum Cardiomyopathy: A Systematic Review of Prospective Studies. Can J Cardiol. 2015;31(12):1421-1426.

21. Hilfiker-Kleiner D, Meyer GP, Schieffer E, et al. Recovery from postpartum cardiomyopathy in 2 patients by blocking prolactin release with bromocriptine. J Am Coll Cardiol. 2012;50(24):2354-2355.

22. Bouabdallaoui N, Mouquet F, Lebreton G, et al. Current knowledge and recent development on management of peripartum cardiomyopathy. Eur Heart J Acute Cardiovasc Care. 2015;doi: 10.1177/2048872615612465.

23. Sheppard R, Rajagopalan N, Safirstein J, et al. An update on treatments and outcomes in peripartum cardiomyopathy. Future Cardiol. 2014;10(3):435-447.

24. Duncker D, Bauersachs J, Hilfiker-Kleiner D, et al. Underestimated risk for life-threatening ventricular arrhythmias in newly diagnosed peripartum cardiomyopathy? Eur Heart J. 2015;36(18):1067-1068.

25. Honigberg MC, Givertz MM. Arrhythmias in peripartum cardiomyopathy. Card Electrophysiol Clin. 2015;7(2):309-317.

26. Dennis AT, Castro JM. Echocardiographic differences between preeclampsia and peripartum cardiomyopathy. Int $J$ Obstet Anesth. 2014;23(3):260-266.

27. Patel H, Madanieh R, Kosmas CE, et al. Reversible Cardiomyopathies. Clin Med Insights Cardiol. 2015;9(Suppl 2):7-14.

28. Shannon-Cain J, Hunt E, Cain BS. Multidisciplinary management of peripartum cardiomyopathy during repeat cesarean delivery: a case report. AANA J. 2008;76(6):443-447.

29. Troponin Reference Range Chang. Peace Health Laboratories. 2013.

30. B-type Natriuretic Peptide (BNP) Blood Test. Cleveland Clinic. 2016.

31. Sliwa K, Förster O, Libhaber E, et al. Peripartum cardiomyopathy: inflammatory markers as predictors of outcome in 100 prospectively studied patients. Eur Heart J. 2006;27(4):441-446.

32. Pearson GD, Veille JC, Rahimtoola S, et al. Peripartum cardiomyopathy: National Heart, Lung, and Blood Institute and Office of Rare Diseases (National Institutes of Health) workshop recommendations and review. JAMA. 2000;283(9):1183-1188.

33. Johnson CT, Hallock JL, Bienstock JL, et al. The John Hopkins Manual of Gynecology and Obstetrics, 5th edn. Wolters Kluwer, Netherlands, 2015:208-211. 